\title{
LOS VALORES DE LOS ADOLESCENTES EN UN CENTRO EDUCATIVO PLURICULTURAL DE CEUTA
}

\section{THE VALUES OF THE TEENAGERS IN A PLURICULTURAL SECONDARY SCHOOL IN CEUTA}

\author{
$M^{a}$ Inmaculada Herrera Ramírez*, Francisco Herrera Clavero**, \\ $M^{a}$ Inmaculada Ramírez Salguero*** \\ Universidad de Granada
}

\section{RESUMEN}

El objetivo de este trabajo era conocer los valores de los adolescentes $(n=200)$ en un IES pluricultural de Ceuta, a través del Cuestionario de Valores - CVH- $\alpha=0,977$ (Herrera, 2007); llegando a las siguientes conclusiones: $1^{\text {a }}$. Los valores más apreciados por los adolescentes son: igualdad, respeto y espíritu de cambio. $2^{\text {a }}$. En función del sexo, las chicas aprecian más la mayoría de los valores analizados. $3^{\mathrm{a}}$. Por curso, destacan los adolescentes de $4^{\mathrm{o}}$ de ESO. $4^{\mathrm{a}}$. Por edad, los de 16 años. $5^{\mathrm{a}}$. Y, en función de sus culturas/religiones, aprecian los valores de distinta forma.

Palabras clave: Curriculum intercultural, Educación en Valores, valores de los adolescentes.

\section{ABSTRACT}

The aim of this research is to know the values of a group of teenagers $(n=200)$ in a multicultural secondary school of Ceuta, applying the Questionnaire of Values - QVH- $\alpha=0,977$ (Herrera, 2007);

* Ma Inmaculada Herrera Ramírez. Maestra, Licenciada en Psicopedagogía y Doctora en Psicopedagogía por la Universidad de Granada. Su Tesis Doctoral versa sobre "Los valores de los adolescentes". Con líneas de investigación en autoconcepto y en valores, y publicaciones en el ámbito del aprendizaje, el currículo, el autoconcepto, los valores, la inmigración, la interculturalidad y la convivencia. Correo-e: iramirez@ugr.es

** Francisco Herrera Clavero. Maestro, doctor en Filosofía y Ciencias de la Educación por la UNED, doctor en Psicología por la Universidad de Granada, catedrático de E.U. en Psicología Evolutiva y de la Educación en la Universidad de Granada, con líneas de investigación y numerosas publicaciones en el ámbito del aprendizaje, el currículo, la inmigración, la interculturalidad y la convivencia, y miembro numerario y de la Junta Rectora del Instituto de Estudios Ceutíes.

*** $M^{a}$ Inmaculada Ramírez Salguero. Maestra, doctora en Filosofía y Ciencias de la Educación por la UNED -Premio Extraordinario-, catedrática de E.U. en Psicología Evolutiva y de la Educación en la Universidad de Granada, con líneas de investigación y numerosas publicaciones en el ámbito del aprendizaje, el currículo, la inmigración, la interculturalidad y la convivencia, y miembro numerario del Instituto de Estudios Ceutíes. 
The following conclusions are drawn: participants most appreciated values are: equality, respect and spirit of change; girls from all grades, 16 years old students and also students from $4^{\text {th }}$ grade show increased appreciation for most of the analyzed values; based on their cultures/religions, teenagers appreciate the values of different form.

Key words: Intercultural curriculum, Education in Values, Values of the teenagers.

\section{Introducción}

El ámbito de los valores ha sido insuficientemente tratado tanto en la educación formal como en la informal y, sin embargo, los valores representan los "faros guía" que iluminan el comportamiento humano, individual y social. Para poder hacernos una mejor idea de a qué nos referimos cuando hablamos sobre "valores" (conceptualización), siendo conscientes de su importancia en la educación, es preciso analizar las definiciones más relevantes. Entre ellas, quizás las más significativas sean las tres que a continuación se ofrecen:

$1^{\mathrm{a}}$. "Los valores son creencias duraderas de que un modo especifico de conducta es personalmente o socialmente preferible a otro contrario o el modo inverso de conducta o estado final de existencia" (Rokeach, 1973, p. 5).

$2^{a}$. "Según la literatura, los valores son (a) conceptos o creencias, (b) sobre estados finales deseables de conductas, (c) que transciende situaciones especificas, (d) de una selección guiada o evaluación de conducta y eventos, y (e) ordenadas por su importancia relativa" (Schwartz y Bilsky, 1987, p. 551).

$3^{\mathrm{a}}$. "Los valores delimitan los parámetros para conductas consideradas aceptables (o justas) y sirven como estructuras para nuestras experiencias" (Hitlin y Piliavin, 2004, p. 363).

Sintéticamente, podríamos concluir que los valores suponen las concepciones, juicios, opiniones, disposiciones y estructuras cognitivas y afectivas, las creencias evaluativos e ideales que sirven de fines para la acción, y los criterios y normas de preferencia que regulan la conducta de las personas y que emanan de su evaluación o de eventos de forma jerárquica, a través de las experiencias vividas.

Nosotros entendemos los valores como disposiciones, creencias y estructuras, cognitivas y afectivas, producto de la experiencia, que actúan a modo de "faros guía" para iluminar, conducir y regular el comportamiento humano, individual y social.

Una vez fijado el concepto sobre los valores, conviene conocer cómo se miden. Hitlin y Piliavin (2004, p. 365) afirman que la medición de los valores, como la de otros muchos conceptos psicológicos y sociales, aún es bastante imperfecta, ya que falta la estandarización entre la investigación teórica y empírica. Los instrumentos más utilizados para medir los valores son los siguientes:

a) La "Rokeach Value Survey" —RVS- (Rokeach, 1967, 1973), escala más conocida para medir valores (en formato ranking), está basada en la distinción entre valores instrumentales y valores terminales, consta de un conjunto de 18 valores instrumentales (modos de conducta: felicidad, libertad, igualdad, placer, reconocimiento social, autoestima, etc.) y otro de 18 valores finales (estados finales de existencia: ambición, honradez, obediencia, limpieza, educación, independencia, etc.), según el grado que los encuestados les concedan como guías principales en 
sus vidas. Rokeach (1973, p. 33) divulgó que las fiabilidades de los valores terminales eran de 0,78 a 0,69 y para los valores instrumentales de 0,71 a 0,61 . No obstante, la identificación de esos 36 valores (instrumentales o terminales) ha sido criticada como arbitraria y subjetiva, y, el propio Rokeach, ha reconocido que su procedimiento fue intuitivo.

b) La "Schwartz Value Survey" - SVS - (Schwartz, 1992, 1994), distingue dos dimensiones de orden superior de valores (en formato rating): Apertura al Cambio frente a Conservación, y Auto-Mejora frente a Auto-Transcendencia, dirigidas en dos sentidos: individualista y colectivista; que se descomponen a su vez en diez tipos motivacionales de valores. Pero, en realidad no mide valores, sino la motivación hacia ellos. Como afirman Hitlin y Piliavin (2004, p. 366), dado que Schwartz no encontró ninguna evidencia empírica para la distinción de Rokeach entre valores instrumentales y terminales, se cuestionó su utilidad, ya que los mismos valores pueden expresar las motivaciones para medios (valores instrumentales) y fines (valores terminales). El análisis estadístico utilizado (Schwartz y Bilsky, 1987, p. 557) partió del cálculo de los coeficientes de correlación Pearson para evaluar la importancia de los valores, coeficientes que sirvieron de matriz de datos para poder llevar a cabo el Análisis de los Espacios más Pequeños (Smallest Space Analisys — SSA — ) de Guttman-Lingoes (1973).

c) Hartman diseñó el Perfil de Valores Hartman (1973), basándose en Rokeach (Hartman Value Profile - HVP-), para su aplicación clínica. No obstante, el Hartman Value Profile (HVP) no mide propiamente los valores personales, sino la capacidad de reconocimiento de los niveles de valor, que son previos a una valoración.

d) El Cuestionario de Valores Personales - SPV - de Gordon (2003), basado en Schwartz, está diseñado para medir el grado o intensidad relativa de cada uno de los seis valores personales propuestos: practicidad, resultados, variedad, decisión, orden y método, y metas o claridad de objetivos a conseguir.

Tras la revisión teórica llevada a cabo, decidimos construir el instrumento sobre valores CVH (Herrera, 2007), por las siguientes razones:

a) Porque ninguno de los instrumentos analizados, a nuestro entender, respondía realmente a lo que queríamos: "analizar los valores de los adolescentes"; y, además, que estos valores fuesen propuestos por ellos mismos.

b) Porque los valores que analizan no son los que nosotros queríamos, ni por su número (nos parecían insuficientes), ni por su orientación (muy subjetivos).

c) Porque queríamos medir sólo valores, empleando un cuestionario especialmente dedicado a ello; pero de los valores que los propios adolescentes y un grupo de expertos (profesores de la Universidad de Granada) identificaran como más comunes y frecuentes, teniendo además en cuenta la Teoría Científica al respecto, y elaborarlo, fiabilizarlo y validarlo, mediante una experiencia piloto que nos permitiera aplicar el cuestionario definitivo.

En suma, este trabajo tiene por objetivo conocer los valores de los adolescentes en un centro educativo pluricultural de Ceuta. 


\section{Método}

\section{Muestra}

El número de participantes en esta investigación ha sido un total de 200 adolescentes de estatus socio-económico medio [renta media mensual $=$ entre 1.500 y $2.000 €(28 \%)$ y nivel de estudios de los padres = EGB (47\%)] y medio bajo [renta media mensual $=$ entre $1.000 \mathrm{y}$ $1.500 €(72 \%)$ y nivel de estudios de los padres = EGB sin terminar (53\%)], alumnos del I.E.S. "Puertas del Campo" de Ceuta en 2006, repartidos de la siguiente forma: 98 varones (49\%) y 102 mujeres (51\%); de edades comprendidas entre los 14 y los 19 años $(14$ años $=10 \%, 15$ años $=25,5 \%, 16$ años $=23,5 \%, 17$ años $=19,5 \%, 18$ años $=16 \%$ y 19 años $=5,5 \%$, ) de los cursos desde $3^{\circ}$ de Enseñanza Secundaria Obligatoria (ESO) hasta $2^{\circ}$ de Bachiller $\left(3^{\circ} \mathrm{ESO}=22 \%, 4^{\circ} \mathrm{ESO}=39,5 \%, 1^{\circ}\right.$ de Bachiller $=15,5 \%$ y $2^{\circ}$ de Bachiller $=23 \%)$; 193 de ellos (96,5\%) nacidos en España y con nacionalidad española y 7 (3,5\%) nacidos en Marruecos y con doble nacionalidad (española y marroquí); y 120 de ellos de cultura/religión marroquí/musulmana (60\%), 68 de la hispano/cristiana (34\%) y el resto ateos o agnósticos $(6 \%)$.

\section{Instrumento y variables}

El instrumento utilizado para la recogida de datos ha sido el Cuestionario de Valores $-\mathrm{CVH}-$, de construcción propia, que trata de obtener información acerca de la apreciación que manifiestan los adolescentes sobre 32 valores, seleccionados a través de una experiencia piloto para recoger las opiniones coincidentes de 51 adolescentes $\left(11\right.$ de $3^{\circ}$ de ESO y 20 de $4^{\circ}$, y 8 de $1^{\circ}$ de Bachiller y 12 de $2^{\circ}$, utilizando el método de muestreo estratificado proporcional) y 3 expertos - jueces externos - (profesores de la Universidad de Granada). La fiabilidad del CVH es de $\alpha=0,977$ (Alfa de Cronbach).

Este instrumento consta de 160 items, a razón de cinco para cada valor, a los que se puede responder otorgándole las siguientes puntuaciones: (1) Totalmente en desacuerdo, (2) En desacuerdo, (3) No tengo opinión, (4) De acuerdo y (5) Totalmente de acuerdo.

El cuestionario recoge las variables contenidas en esta investigación, clasificadas de la siguiente forma:

a) Variables de estudio: Amistad, amor, bondad, civismo, cooperación, democracia, diálogo, esfuerzo, espíritu de cambio, ética, familia, generosidad, honradez, humanidad, ideas y creencias de nuestros mayores, igualdad, justicia, legalidad, libertad, liderazgo, moralidad, motivación de logro, paz, prudencia, religión, respeto, solidaridad, superación, tiempo libre y ocio, tolerancia, trabajo y verdad.

b) Variables de clasificación: Sexo, edad, curso, estatus socio-económico familiar, país de nacimiento y nacionalidad, cultura/religión.

\section{Procedimiento}

Los autores aplicaron el CVH en el IES "Puertas del Campo" de Ceuta en abril de 2007, en sesiones de una hora y en días diferentes, para cada curso. 
Para ello, respetando escrupulosamente las normas deontológicas, se les repartió, presentó y explicó a los adolescentes el CVH, indicándoles que el cuestionario era anónimo y confidencial, y que pretendía recoger sus opiniones acerca de 32 valores, en función de la importancia que les concedían en sus vidas, rogándoles que contestaran con sinceridad, otorgándoles las puntuaciones que creyeran más oportunas en cada caso, de las cinco posibles que se les ofrecían: 1. Totalmente de desacuerdo, 2. Algo de desacuerdo, 3. No tengo opinión formada, 4. Algo de acuerdo, 5. Totalmente de acuerdo.

\section{Análisis estadístico}

Para el análisis estadístico de los datos recogidos se utilizó el SPSS, versión 14.0 para Windows, aplicado en dos niveles:

$1^{\circ}$. Descriptivo. Para facilitar el conocimiento de las características del estudio -estadísticos notables-, analizando las propiedades más relevantes que sirvieron como punto de partida para los análisis posteriores. Con tal fin, se utilizaron las medidas de tendencia central, (promedios — media, mediana y moda-, representativas del conjunto de la distribución y las de variabilidad (dispersión —-desviación típica y varianza-, simetría y curtosis), para saber cómo se agrupaban los datos. Asimismo, se aplicaron las pruebas de normalidad (Kolmogorov-Smirnov) y de bondad de ajuste (binomial, para las variables cualitativas de dos alternativas, y la prueba $\mathrm{Ji}^{2}\left(\mathrm{chi}^{2}\right)$, para las variables cualitativas de más de dos alternativas y las variables cuantitativas).

$2^{\circ}$. Inferencial. La estadística inferencial sirvió para delimitar qué diferencias de las halladas se debían al azar y cuáles no, utilizando pruebas "U" de MannWhitney y "W" de Wilcoxon, para dos muestras independientes de los valores de los adolescentes, con las variable de agrupación "sexo", "estatus socio-económico familiar" y "cultura/religión"; el ANOVA de un solo factor de los valores de los adolescentes, con la variable de agrupación "edad"; y la prueba de Kruskal Wallis para dos muestras independientes de los valores de los adolescentes, con las variables de agrupación "curso" y "país de nacimiento y nacionalidad".

Asimismo, se utilizaron el Análisis de Fiabilidad (Alfa de Cronbach) y el Análisis Factorial (Método de componentes principales, con rotación varimax), para hallar la fiabilidad y la validez del instrumento.

\section{Resultados}

Los resultados obtenidos fueron los siguientes:

$1^{\circ}$. Los valores más apreciados por los adolescentes de la muestra, ordenados de mayor a menor, en función de las medias aritméticas y sus correspondientes desviaciones típicas obtenidas de sus respuestas, son los que recoge la tabla 1; destacando como los 10 valores más apreciados: igualdad, respeto, espíritu de cambio, verdad, familia, diálogo, paz, bondad, justicia, generosidad. 
Los valores de los adolescentes en un centro educativo ...

Ma Inmaculada Herrera Ramírez et al.

TABLA 1: Valores más apreciados por los adolescentes de la muestra, de mayor a menor, en función de sus medias aritméticas y desviaciones típicas.

\begin{tabular}{|c|c|c|c|c|c|c|c|c|}
\hline Global & $x$ & $\bar{x}$ & Musulmanes & $\overline{\mathbf{x}}$ & $\delta$ & Cristianos & $\overline{\mathbf{x}}$ & $\delta$ \\
\hline Igualdad & 22,62 & 2,59 & Igualdad & 22,92 & 2,55 & Familia & 22,66 & 2,95 \\
\hline Respeto & 22,16 & 2,90 & Verdad & 22,79 & 2,97 & Respeto & 22,62 & 2,16 \\
\hline $\begin{array}{l}\text { Espíritu de } \\
\text { cambio }\end{array}$ & 22,05 & 2,48 & Diálogo & 22,33 & 3,21 & Esfuerzo & 22,41 & 2,57 \\
\hline Verdad & 22,03 & 3,41 & Bondad & 22,08 & 3,02 & Amistad & 22,34 & 1,90 \\
\hline Familia & 22,03 & 4,27 & Justicia & 22,06 & 2,60 & $\begin{array}{l}\text { Espíritu de } \\
\text { cambio }\end{array}$ & 22,25 & 2,51 \\
\hline Diálogo & 21,99 & 3,548 & Generosidad & 22,05 & 2,73 & Paz & 22,19 & 3,26 \\
\hline Paz & 21,90 & 3,64 & $\begin{array}{l}\text { Espíritu de } \\
\text { cambio }\end{array}$ & 22,00 & 2,55 & Igualdad & 22,07 & 2,61 \\
\hline Bondad & 21,84 & 2,95 & Humanidad & 21,98 & 3,63 & Diálogo & 21,98 & 3,50 \\
\hline Justicia & 21,77 & 2,50 & Respeto & 21,97 & 3,21 & Bondad & 21,87 & 2,80 \\
\hline Generosidad & 21,64 & 3,31 & Familia & 21,83 & 4,82 & $\begin{array}{l}\text { Tiempo libre } \\
\text { y ocio }\end{array}$ & 21,82 & 2,25 \\
\hline Humanidad & 21,50 & 3,55 & Paz & 21,79 & 3,81 & Civismo & 21,70 & 3,00 \\
\hline $\begin{array}{l}\text { Tiempo libre } \\
\text { y ocio }\end{array}$ & 21,42 & 2,63 & Solidaridad & 21,71 & 3,73 & Generosidad & 21,69 & 3,78 \\
\hline Esfuerzo & 21,44 & 3,50 & Ética & 21,40 & 3,45 & Ética & 21,65 & 3,16 \\
\hline Amistad & 21,39 & 2,61 & Civismo & 21,37 & 3,55 & Democracia & 21,59 & 2,60 \\
\hline Ética & 21,30 & 3,40 & Prudencia & 21,32 & 3,14 & Justicia & 21,50 & 2,29 \\
\hline Legalidad & 21,28 & 3,04 & Legalidad & 21,22 & 2,90 & Humanidad & 21,41 & 3,11 \\
\hline Democracia & 21,23 & 2,88 & Libertad & 21,16 & 3,09 & Honradez & 21,37 & 3,03 \\
\hline Civismo & 21,17 & 3,55 & $\begin{array}{l}\text { Tiempo libre } \\
\text { y ocio }\end{array}$ & 21,15 & 2,90 & Legalidad & 21,34 & 3,43 \\
\hline Solidaridad & 21,10 & 3,70 & $\begin{array}{l}\text { Motivación de } \\
\text { logro }\end{array}$ & 21,10 & 2,78 & Verdad & 21,25 & 3,67 \\
\hline Libertad & 21,08 & 2,91 & Democracia & 21,00 & 3,04 & Trabajo & 21,07 & 3,62 \\
\hline Prudencia & 20,95 & 3,21 & Esfuerzo & 20,82 & 3,84 & Solidaridad & 20,91 & 3,04 \\
\hline Trabajo & 20,85 & 3,30 & Amistad & 20,80 & 2,85 & Libertad & 20,56 & 2,53 \\
\hline $\begin{array}{l}\text { Motivación de } \\
\text { logro }\end{array}$ & 20,82 & 2,74 & Religión & 20,77 & 3,99 & $\begin{array}{l}\text { Motivación de } \\
\text { logro }\end{array}$ & 20,45 & 2,13 \\
\hline
\end{tabular}


$M^{a}$ Inmaculada Herrera Ramírez et al. Los valores de los adolescentes en un centro educativo ...

\begin{tabular}{|l|c|c|l|c|c|l|c|c|}
\hline \multicolumn{1}{|c|}{ Global } & $\mathbf{X}$ & $\overline{\mathbf{X}}$ & Musulmanes & $\overline{\mathbf{X}}$ & $\boldsymbol{\delta}$ & Cristianos & $\overline{\mathbf{X}}$ & $\boldsymbol{\delta}$ \\
\hline Honradez & 20,68 & 3,50 & Trabajo & 20,68 & 3,19 & Prudencia & 20,43 & 3,26 \\
\hline $\begin{array}{l}\text { Ideas de los } \\
\text { mayores }\end{array}$ & 20,35 & 3,67 & $\begin{array}{l}\text { Ideas de los } \\
\text { mayores }\end{array}$ & 20,67 & 3,36 & Cooperación & 20,28 & 3,67 \\
\hline Tolerancia & 20,17 & 3,19 & Amor & 20,47 & 3,76 & Superación & 20,10 & 3,97 \\
\hline Amor & 20,08 & 3,40 & Honradez & 20,43 & 3,80 & Tolerancia & 19,97 & 2,30 \\
\hline Superación & 19,99 & 4,28 & Cooperación & 20,38 & 3,88 & Amor & 19,78 & 2,60 \\
\hline Cooperación & 19,98 & 4,05 & Tolerancia & 20,27 & 3,72 & $\begin{array}{l}\text { Ideas de los } \\
\text { mayores }\end{array}$ & 19,66 & 4,17 \\
\hline Moralidad & 19,34 & 3,65 & Superación & 19,79 & 4,40 & Moralidad & 19,19 & 3,58 \\
\hline Religión & 19,08 & 5,03 & Moralidad & 19,34 & 3,71 & Religión & 17,63 & 4,91 \\
\hline Liderazgo & 16,72 & 3,30 & Liderazgo & 17,62 & 2,92 & Liderazgo & 15,12 & 3,44 \\
\hline
\end{tabular}

$2^{\circ}$. En función del "sexo" (tabla 2), aplicadas las pruebas "U" de Mann-Whitney y "W" de Wilcoxon, para dos muestras independientes, a los valores de los adolescentes, se han hallado diferencias estadísticamente significativas en la mayoría de los valores analizados, obteniendo las adolescentes los mayores rangos promedio en: amistad (p. 0,001), amor (p. 0,000), cooperación (p. 0,038), espíritu de cambio (p. 0,020), familia (p. 0,010), generosidad (p. 0,000), honradez (p. 0,033), humanidad (p. 0,001), igualdad (p. 0,007), legalidad (p. 0,020), libertad (p. 0,007), paz (p. 0,029), respeto (p. 0,000), superación (p. 0,001), tiempo libre y ocio (p. 0,000), tolerancia (p. 0,015$)$ y verdad (p. 0,013); mientras que los adolescentes sólo en liderazgo (p. 0,028). En el resto de los valores analizados, los adolescentes de ambos sexos comparten la misma apreciación.

TABLA 2: Pruebas "U" de Mann-Whitney y "W" de Wilcoxon, para dos muestras independientes de los valores de los adolescentes, con la variable de agrupación "sexo".

\begin{tabular}{|c|c|c|c|c|c|c|c|}
\hline Valores & Sexo & $N$ & U & $\begin{array}{c}\text { Rango } \\
\text { Promedio }\end{array}$ & $\mathbf{w}$ & $\mathbf{z}$ & $\mathbf{P}$ \\
\hline Amistad & $\begin{array}{l}\text { Hombre } \\
\text { Mujer }\end{array}$ & $\begin{array}{r}98 \\
102\end{array}$ & $3.684,5$ & $\begin{array}{r}87,10 \\
113,38\end{array}$ & $8.535,5$ & $-3,245$ & ,001 \\
\hline Amor & $\begin{array}{l}\text { Hombre } \\
\text { Mujer }\end{array}$ & $\begin{array}{r}98 \\
102\end{array}$ & $3.441,5$ & $\begin{array}{r}84,62 \\
115,76\end{array}$ & $8.292,5$ & $-3,826$ & ,000 \\
\hline Cooperación & $\begin{array}{l}\text { Hombre } \\
\text { Mujer }\end{array}$ & $\begin{array}{r}98 \\
102\end{array}$ & $4.156,5$ & $\begin{array}{r}91,91 \\
108,75\end{array}$ & $9.007,5$ & $-2,071$ & ,038 \\
\hline $\begin{array}{l}\text { Espíritu de } \\
\text { cambio }\end{array}$ & $\begin{array}{l}\text { Hombre } \\
\text { Mujer }\end{array}$ & $\begin{array}{r}98 \\
102\end{array}$ & $4.055,5$ & $\begin{array}{r}90,88 \\
109,74\end{array}$ & $8.906,5$ & $-2,331$ & ,020 \\
\hline Familia & $\begin{array}{l}\text { Hombre } \\
\text { Mujer }\end{array}$ & $\begin{array}{r}98 \\
102\end{array}$ & $3.977,5$ & $\begin{array}{r}90,09 \\
110,50\end{array}$ & $8.828,5$ & $-2,588$ & ,010 \\
\hline
\end{tabular}




\begin{tabular}{|c|c|c|c|c|c|c|c|}
\hline Valores & Sexo & $\mathbf{N}$ & U & $\begin{array}{c}\text { Rango } \\
\text { Promedio }\end{array}$ & $\mathbf{w}$ & $\mathbf{z}$ & $\mathbf{P}$ \\
\hline Generosidad & $\begin{array}{l}\text { Hombre } \\
\text { Mujer }\end{array}$ & $\begin{array}{r}98 \\
102\end{array}$ & $3.370,5$ & $\begin{array}{r}83,89 \\
116,46\end{array}$ & $8.221,5$ & $-4,045$ & ,000 \\
\hline Honradez & $\begin{array}{l}\text { Hombre } \\
\text { Mujer }\end{array}$ & $\begin{array}{r}98 \\
102\end{array}$ & $4.131,0$ & $\begin{array}{r}91,65 \\
109,00\end{array}$ & $8.982,0$ & $-2,134$ & ,033 \\
\hline Humanidad & $\begin{array}{l}\text { Hombre } \\
\text { Mujer }\end{array}$ & $\begin{array}{r}98 \\
102\end{array}$ & $3.696,5$ & $\begin{array}{r}87,22 \\
113,26\end{array}$ & $8.547,5$ & $-3,222$ & ,001 \\
\hline Igualdad & $\begin{array}{l}\text { Hombre } \\
\text { Mujer }\end{array}$ & $\begin{array}{r}98 \\
102\end{array}$ & $3.915,0$ & $\begin{array}{r}89,45 \\
111,12\end{array}$ & $8.766,0$ & $-2,711$ & ,007 \\
\hline Legalidad & $\begin{array}{l}\text { Hombre } \\
\text { Mujer }\end{array}$ & $\begin{array}{r}98 \\
102\end{array}$ & $4.055,0$ & $\begin{array}{r}90,88 \\
109,75\end{array}$ & $8.906,0$ & $-2,322$ & ,020 \\
\hline Libertad & $\begin{array}{l}\text { Hombre } \\
\text { Mujer }\end{array}$ & $\begin{array}{r}98 \\
102\end{array}$ & $3.916,5$ & $\begin{array}{r}89,46 \\
111,10\end{array}$ & $8.767,5$ & $-2,676$ & ,007 \\
\hline Liderazgo & $\begin{array}{l}\text { Hombre } \\
\text { Mujer }\end{array}$ & $\begin{array}{r}98 \\
102\end{array}$ & $4.103,5$ & $\begin{array}{r}109,63 \\
91,73\end{array}$ & $9.356,5$ & $-2,203$ & ,028 \\
\hline Paz & $\begin{array}{l}\text { Hombre } \\
\text { Mujer }\end{array}$ & $\begin{array}{r}98 \\
102\end{array}$ & $4.135,0$ & $\begin{array}{r}91,69 \\
108,96\end{array}$ & $8.986,0$ & $-2,184$ & ,029 \\
\hline Respeto & $\begin{array}{l}\text { Hombre } \\
\text { Mujer }\end{array}$ & $\begin{array}{r}98 \\
102\end{array}$ & $3.114,0$ & $\begin{array}{r}81,28 \\
118,97\end{array}$ & $7.965,0$ & $-4,669$ & ,000 \\
\hline Superación & $\begin{array}{l}\text { Hombre } \\
\text { Mujer }\end{array}$ & $\begin{array}{r}98 \\
102\end{array}$ & $3.604,5$ & $\begin{array}{r}86,28 \\
114,16\end{array}$ & $8.455,5$ & $-3,428$ & ,001 \\
\hline $\begin{array}{l}\text { Tiempo libre y } \\
\text { ocio }\end{array}$ & $\begin{array}{l}\text { Hombre } \\
\text { Mujer }\end{array}$ & $\begin{array}{r}98 \\
102\end{array}$ & $3.560,0$ & $\begin{array}{r}85,83 \\
114,60\end{array}$ & $8.411,0$ & $-3,576$ & ,000 \\
\hline Tolerancia & $\begin{array}{l}\text { Hombre } \\
\text { Mujer }\end{array}$ & $\begin{array}{r}98 \\
102\end{array}$ & $4.010,0$ & $\begin{array}{r}90,42 \\
110,19\end{array}$ & $8.861,0$ & $-2,437$ & ,015 \\
\hline Verdad & $\begin{array}{l}\text { Hombre } \\
\text { Mujer }\end{array}$ & $\begin{array}{r}98 \\
102\end{array}$ & $4.001,0$ & $\begin{array}{r}90,33 \\
110,27\end{array}$ & $8.852,0$ & $-2,496$ & ,013 \\
\hline
\end{tabular}

$3^{\circ}$. En función de la "edad" (tabla 3), a través del ANOVA de un solo factor de los valores de los adolescentes, se han encontrado diferencias estadísticamente significativas en los siguientes: amor (p. 0,000), bondad (p. 0,005), civismo (p. 0,001), cooperación (p. 0,000), diálogo (p. 0,004), espíritu de cambio (p. 0,002), ética (p. 0,003), generosidad (p. 0,000), honradez (p. 0,022), ideas de los mayores (p. 0,000), justicia (p. 0,000), liderazgo (p. 0,005), moralidad (p. 0,024), prudencia (p. 0,000), religión (p. 0,000), trabajo (p. 0,000) y verdad (p. 0,000). Tras el análisis post hoc (prueba DMS), se ha podido comprobar que las diferencias halladas destacan a los adolescentes de 16 años en: amistad (p. 0,033), amor (p. 0,018), bondad (p. 0,000), civismo (p. 0,000), cooperación (p. 0,002), diálogo (p. 0,007), espíritu de cambio (p. 0,004), ética (p. 0,004), generosidad (p. 0,000), honradez (p. 0,019), ideas de los mayores (p. 0,028), justicia (p. 0,000), legalidad (p. 0,023), 
$M^{a}$ Inmaculada Herrera Ramírez et al. Los valores de los adolescentes en un centro educativo ...

motivación de logro (p. 0,005), paz (p. 0,005), prudencia (p. 0,000), religión (p. 0,004), respeto (p. 0,010), solidaridad (p. 0,019), superación (p. 0,023), trabajo (p. 0,000) y verdad (p. 0,000); a los de 15 años en: democracia (p. 0,014), humanidad (p. 0,029), liderazgo (p. 0,028) y tolerancia (p. 0,046); y a los de 18 años en: esfuerzo (p. 0,007), moralidad (p. 0,020) y tiempo libre y ocio (p. 0,031).

TABLA 3: ANOVA de un solo factor de los valores de los adolescentes, con la variable de agrupación "edad".

\begin{tabular}{|c|c|c|c|c|c|c|}
\hline Valores & & $\begin{array}{l}\text { Suma de } \\
\text { Cuadrados }\end{array}$ & G.L. & $\begin{array}{c}\text { Media } \\
\text { Cuadrática }\end{array}$ & $\mathbf{F}$ & $\mathbf{P}$ \\
\hline Amor & $\begin{array}{l}\text { Inter-grupos } \\
\text { Intra-grupos } \\
\text { Total }\end{array}$ & $\begin{array}{r}249,575 \\
2.048,300 \\
2.297,875\end{array}$ & $\begin{array}{r}5 \\
195 \\
200\end{array}$ & $\begin{array}{l}49,915 \\
10,558\end{array}$ & 4,728 & ,000 \\
\hline Bondad & $\begin{array}{l}\text { Inter-grupos } \\
\text { Intra-grupos } \\
\text { Total }\end{array}$ & $\begin{array}{r}134,560 \\
1.602,995 \\
1.737,555\end{array}$ & $\begin{array}{r}5 \\
195 \\
200\end{array}$ & $\begin{array}{r}26,912 \\
8,263\end{array}$ & 3,257 & ,005 \\
\hline Civismo & $\begin{array}{l}\text { Inter-grupos } \\
\text { Intra-grupos } \\
\text { Total }\end{array}$ & $\begin{array}{r}256,204 \\
2.247,351 \\
2.503,555\end{array}$ & $\begin{array}{r}5 \\
195 \\
200\end{array}$ & $\begin{array}{l}51,241 \\
11,584\end{array}$ & 4,423 & ,001 \\
\hline Cooperación & $\begin{array}{l}\text { Inter-grupos } \\
\text { Intra-grupos } \\
\text { Total }\end{array}$ & $\begin{array}{r}451,693 \\
2.820,227 \\
3.271,920\end{array}$ & $\begin{array}{r}5 \\
195 \\
200\end{array}$ & $\begin{array}{l}90,339 \\
14,537\end{array}$ & 6,214 & ,000 \\
\hline Diálogo & $\begin{array}{l}\text { Inter-grupos } \\
\text { Intra-grupos } \\
\text { Total }\end{array}$ & $\begin{array}{r}213,631 \\
2.291,364 \\
2.504,995\end{array}$ & $\begin{array}{r}5 \\
195 \\
200\end{array}$ & $\begin{array}{l}42,726 \\
11,811\end{array}$ & 3,617 & ,004 \\
\hline $\begin{array}{l}\text { Espíritu de } \\
\text { cambio }\end{array}$ & $\begin{array}{l}\text { Inter-grupos } \\
\text { Intra-grupos } \\
\text { Total }\end{array}$ & $\begin{array}{r}112,988 \\
1.112,512 \\
1.225,500\end{array}$ & $\begin{array}{r}5 \\
195 \\
200\end{array}$ & $\begin{array}{r}22,598 \\
5,735\end{array}$ & 3,941 & ,002 \\
\hline Ética & $\begin{array}{l}\text { Inter-grupos } \\
\text { Intra-grupos } \\
\text { Total }\end{array}$ & $\begin{array}{r}198,891 \\
2.104,704 \\
2.303,595\end{array}$ & $\begin{array}{r}5 \\
195 \\
200\end{array}$ & $\begin{array}{l}39,778 \\
10,849\end{array}$ & 3,667 & ,003 \\
\hline Generosidad & $\begin{array}{l}\text { Inter-grupos } \\
\text { Intra-grupos } \\
\text { Total }\end{array}$ & $\begin{array}{r}254,302 \\
1.931,778 \\
2.186,080\end{array}$ & $\begin{array}{r}5 \\
195 \\
200\end{array}$ & $\begin{array}{r}50,860 \\
9,958\end{array}$ & 5,108 & ,000 \\
\hline Honradez & $\begin{array}{l}\text { Inter-grupos } \\
\text { Intra-grupos } \\
\text { Total }\end{array}$ & $\begin{array}{r}158,925 \\
2.276,595 \\
2.435,520\end{array}$ & $\begin{array}{r}5 \\
195 \\
200\end{array}$ & $\begin{array}{l}31,785 \\
11,735\end{array}$ & 2,709 & ,022 \\
\hline $\begin{array}{l}\text { Ideas de los } \\
\text { mayores }\end{array}$ & $\begin{array}{l}\text { Inter-grupos } \\
\text { Intra-grupos } \\
\text { Total }\end{array}$ & $\begin{array}{r}298,474 \\
2.386,721 \\
2.685,195\end{array}$ & $\begin{array}{r}5 \\
195 \\
200\end{array}$ & $\begin{array}{l}59,695 \\
12,303\end{array}$ & 4,852 & ,000 \\
\hline Justicia & $\begin{array}{l}\text { Inter-grupos } \\
\text { Intra-grupos } \\
\text { Total }\end{array}$ & $\begin{array}{r}162,572 \\
1.080,848 \\
1.243,420\end{array}$ & $\begin{array}{r}5 \\
195 \\
200\end{array}$ & $\begin{array}{r}32,514 \\
5,571\end{array}$ & 5,836 & ,000 \\
\hline
\end{tabular}




\begin{tabular}{|c|c|c|c|c|c|c|}
\hline Valores & & $\begin{array}{l}\text { Suma de } \\
\text { Cuadrados }\end{array}$ & G.L. & $\begin{array}{c}\text { Media } \\
\text { Cuadrática }\end{array}$ & $\mathbf{F}$ & $\mathbf{P}$ \\
\hline Liderazgo & $\begin{array}{l}\text { Inter-grupos } \\
\text { Intra-grupos } \\
\text { Total }\end{array}$ & $\begin{array}{r}175,196 \\
1.993,124 \\
2.168,320\end{array}$ & $\begin{array}{r}5 \\
195 \\
200\end{array}$ & $\begin{array}{l}35,039 \\
10,274\end{array}$ & 3,411 & ,005 \\
\hline Moralidad & $\begin{array}{l}\text { Inter-grupos } \\
\text { Intra-grupos } \\
\text { Total }\end{array}$ & $\begin{array}{r}170,247 \\
2.482,633 \\
2.652,880\end{array}$ & $\begin{array}{r}5 \\
195 \\
200\end{array}$ & $\begin{array}{l}34,049 \\
12,797\end{array}$ & 2,661 & ,024 \\
\hline Prudencia & $\begin{array}{l}\text { Inter-grupos } \\
\text { Intra-grupos } \\
\text { Total }\end{array}$ & $\begin{array}{r}253,674 \\
1.799,826 \\
2.053,500\end{array}$ & $\begin{array}{r}5 \\
195 \\
200\end{array}$ & $\begin{array}{r}50,735 \\
9,277\end{array}$ & 5,469 & ,000 \\
\hline Religión & $\begin{array}{l}\text { Inter-grupos } \\
\text { Intra-grupos } \\
\text { Total }\end{array}$ & $\begin{array}{r}903,580 \\
4.128,295 \\
5.031,875\end{array}$ & $\begin{array}{r}5 \\
195 \\
200\end{array}$ & $\begin{array}{r}180,716 \\
21,280\end{array}$ & 8,492 & ,000 \\
\hline Trabajo & $\begin{array}{l}\text { Inter-grupos } \\
\text { Intra-grupos } \\
\text { Total }\end{array}$ & $\begin{array}{r}270,209 \\
1.893,291 \\
2.163,500\end{array}$ & $\begin{array}{r}5 \\
195 \\
200\end{array}$ & $\begin{array}{r}54,042 \\
9,759\end{array}$ & $\begin{array}{l}5,537 \\
1,678\end{array}$ & ,000 \\
\hline Verdad & $\begin{array}{l}\text { Inter-grupos } \\
\text { Intra-grupos } \\
\text { Total }\end{array}$ & $\begin{array}{c}56,283 \\
1.301,072 \\
1.357,355\end{array}$ & $\begin{array}{r}5 \\
195 \\
200\end{array}$ & $\begin{array}{l}57,992 \\
10,438\end{array}$ & 4,728 & ,000 \\
\hline
\end{tabular}

$4^{\circ}$. En función del "curso" (tabla 4), aplicada la prueba de Kruskal Wallis para "k" muestras independientes a los valores de los adolescentes, se han hallado diferencias estadísticamente significativas en los siguientes valores analizados: amor (p. 0,022), bondad (p. 0,000), civismo (p. 0,000), cooperación (p. 0,000), democracia (p. 0,000), diálogo (p. 0,000), espíritu de cambio (p. 0,000), ética (p. 0,000), generosidad (p. 0,000), honradez (p. 0,000), humanidad (p. 0,000), ideas de los mayores (p. 0,000), justicia (p. 0,000), legalidad (p. 0,000), libertad (p. 0,000), liderazgo (p. 0,000), moralidad (p. 0,000), paz (p. 0,000), prudencia (p. 0,000), religión (p. 0,000), respeto (p. 0,000), solidaridad (p. 0,000), trabajo (p. 0,004) y verdad (p. 0,000); obteniendo siempre los mayores rangos promedio los adolescentes de $4^{\circ}$ de ESO.

TABLA 4: Prueba de Kruskal Wallis para dos muestras independientes de los valores de los adolescentes, con la variable de agrupación "curso".

\begin{tabular}{|c|c|c|c|c|c|c|}
\hline Valores & Curso & $\mathbf{N}$ & Rango Promedio & G.L. & $\mathrm{Ji}^{2}$ & $\mathbf{P}$ \\
\hline Amor & $\begin{array}{l}3^{\circ} \text { ESO } \\
4^{\circ} \text { ESO } \\
1^{\circ} \text { Bachiller } \\
2^{\circ} \text { Bachiller }\end{array}$ & $\begin{array}{l}44 \\
79 \\
31 \\
46\end{array}$ & $\begin{array}{r}81,99 \\
114,10 \\
101,90 \\
93,90\end{array}$ & 3 & 9,592 & ,022 \\
\hline Bondad & $\begin{array}{l}3^{\circ} \text { ESO } \\
4^{\circ} \text { ESO } \\
1^{\circ} \text { Bachiller } \\
2^{\circ} \text { Bachiller }\end{array}$ & $\begin{array}{l}44 \\
79 \\
31 \\
46\end{array}$ & $\begin{array}{r}71,34 \\
127,53 \\
82,06 \\
94,39\end{array}$ & 3 & 32,847 & ,000 \\
\hline
\end{tabular}


$M^{a}$ Inmaculada Herrera Ramírez et al. Los valores de los adolescentes en un centro educativo ...

\begin{tabular}{|c|c|c|c|c|c|c|}
\hline Valores & Curso & $\mathbf{N}$ & Rango Promedio & G.L. & $\mathrm{Ji}^{2}$ & $\mathbf{P}$ \\
\hline Civismo & $\begin{array}{l}3^{\circ} \text { ESO } \\
4^{\circ} \text { ESO } \\
1^{\circ} \text { Bachiller } \\
2^{\circ} \text { Bachiller }\end{array}$ & $\begin{array}{l}44 \\
79 \\
31 \\
46\end{array}$ & $\begin{array}{r}67,58 \\
127,96 \\
90,98 \\
91,25\end{array}$ & 3 & 34,621 &, 000 \\
\hline Cooperación & $\begin{array}{l}3^{\circ} \text { ESO } \\
4^{\circ} \text { ESO } \\
1^{\circ} \text { Bachiller } \\
2^{\circ} \text { Bachiller }\end{array}$ & $\begin{array}{l}44 \\
79 \\
31 \\
46\end{array}$ & $\begin{array}{r}79,81 \\
121,91 \\
99,89 \\
83,93\end{array}$ & 3 & 20,491 &, 000 \\
\hline Democracia & $\begin{array}{l}3^{\circ} \text { ESO } \\
4^{\circ} \text { ESO } \\
1^{\circ} \text { Bachiller } \\
2^{\circ} \text { Bachiller }\end{array}$ & $\begin{array}{l}44 \\
79 \\
31 \\
46\end{array}$ & $\begin{array}{r}73,74 \\
126,10 \\
93,77 \\
86,66\end{array}$ & 3 & 28,451 &, 000 \\
\hline Diálogo & $\begin{array}{l}3^{\circ} \text { ESO } \\
4^{\circ} \text { ESO } \\
1^{\circ} \text { Bachiller } \\
2^{\circ} \text { Bachiller }\end{array}$ & $\begin{array}{l}44 \\
79 \\
31 \\
46\end{array}$ & $\begin{array}{r}74,25 \\
127,47 \\
87,98 \\
87,72\end{array}$ & 3 & 31,166 & ,000 \\
\hline $\begin{array}{l}\text { Espíritu de } \\
\text { cambio }\end{array}$ & $\begin{array}{l}3^{\circ} \text { ESO } \\
4^{\circ} \text { ESO } \\
1^{\circ} \text { Bachiller } \\
2^{\circ} \text { Bachiller }\end{array}$ & $\begin{array}{l}44 \\
79 \\
31 \\
46\end{array}$ & $\begin{array}{r}79,65 \\
121,65 \\
82,35 \\
96,36\end{array}$ & 3 & 20,008 &, 000 \\
\hline Ética & $\begin{array}{l}3^{\circ} \text { ESO } \\
4^{\circ} \text { ESO } \\
1^{\circ} \text { Bachiller } \\
2^{\circ} \text { Bachiller }\end{array}$ & $\begin{array}{l}44 \\
79 \\
31 \\
46\end{array}$ & $\begin{array}{r}74,49 \\
123,15 \\
89,97 \\
93,58\end{array}$ & 3 & 23,102 &, 000 \\
\hline Generosidad & $\begin{array}{l}3^{\circ} \text { ESO } \\
4^{\circ} \text { ESO } \\
1^{\circ} \text { Bachiller } \\
2^{\circ} \text { Bachiller }\end{array}$ & $\begin{array}{l}44 \\
79 \\
31 \\
46\end{array}$ & $\begin{array}{r}68,98 \\
124,09 \\
92,44 \\
95,57\end{array}$ & 3 & 28,043 &, 000 \\
\hline Honradez & $\begin{array}{l}3^{\circ} \text { ESO } \\
4^{\circ} \text { ESO } \\
1^{\circ} \text { Bachiller } \\
2^{\circ} \text { Bachiller }\end{array}$ & $\begin{array}{l}44 \\
79 \\
31 \\
46\end{array}$ & $\begin{array}{r}79,89 \\
125,33 \\
86,42 \\
87,07\end{array}$ & 3 & 24,776 & ,000 \\
\hline Humanidad & $\begin{array}{l}3^{\circ} \text { ESO } \\
4^{\circ} \text { ESO } \\
1^{\circ} \text { Bachiller } \\
2^{\circ} \text { Bachiller }\end{array}$ & $\begin{array}{l}44 \\
79 \\
31 \\
46\end{array}$ & $\begin{array}{r}86,08 \\
126,22 \\
76,69 \\
86,16\end{array}$ & 3 & 27,096 &, 000 \\
\hline $\begin{array}{l}\text { Ideas de los } \\
\text { mayores }\end{array}$ & $\begin{array}{l}3^{\circ} \text { ESO } \\
4^{\circ} \text { ESO } \\
1^{\circ} \text { Bachiller } \\
2^{\circ} \text { Bachiller }\end{array}$ & $\begin{array}{l}44 \\
79 \\
31 \\
46\end{array}$ & $\begin{array}{r}83,92 \\
122,49 \\
72,61 \\
97,39\end{array}$ & 3 & 22,601 &, 000 \\
\hline Justicia & $\begin{array}{l}3^{\circ} \text { ESO } \\
4^{\circ} \text { ESO } \\
1^{\circ} \text { Bachiller } \\
2^{\circ} \text { Bachiller }\end{array}$ & $\begin{array}{l}44 \\
79 \\
31 \\
46\end{array}$ & $\begin{array}{r}69,63 \\
132,55 \\
85,66 \\
84,99\end{array}$ & 3 & 43,185 &, 000 \\
\hline
\end{tabular}


Los valores de los adolescentes en un centro educativo ... $M^{a}$ Inmaculada Herrera Ramírez et al.

\begin{tabular}{|c|c|c|c|c|c|c|}
\hline Valores & Curso & $\mathbf{N}$ & Rango Promedio & G.L. & $\mathrm{Ji}^{2}$ & $\mathbf{P}$ \\
\hline Legalidad & $\begin{array}{l}3^{\circ} \text { ESO } \\
4^{\circ} \text { ESO } \\
1^{\circ} \text { Bachiller } \\
2^{\circ} \text { Bachiller }\end{array}$ & $\begin{array}{l}44 \\
79 \\
31 \\
46\end{array}$ & $\begin{array}{r}88,80 \\
121,08 \\
80,21 \\
90,02\end{array}$ & 3 & 17,361 & ,001 \\
\hline Libertad & $\begin{array}{l}3^{\circ} \text { ESO } \\
4^{\circ} \text { ESO } \\
1^{\circ} \text { Bachiller } \\
2^{\circ} \text { Bachiller }\end{array}$ & $\begin{array}{l}44 \\
79 \\
31 \\
46\end{array}$ & $\begin{array}{r}91,56 \\
118,58 \\
80,08 \\
91,76\end{array}$ & 3 & 14,009 & ,003 \\
\hline Liderazgo & $\begin{array}{l}3^{\circ} \text { ESO } \\
4^{\circ} \text { ESO } \\
1^{\circ} \text { Bachiller } \\
2^{\circ} \text { Bachiller }\end{array}$ & $\begin{array}{l}44 \\
79 \\
31 \\
46\end{array}$ & $\begin{array}{r}109,86 \\
115,00 \\
66,26 \\
89,72\end{array}$ & 3 & 18,848 & ,000 \\
\hline Moralidad & $\begin{array}{l}3^{\circ} \text { ESO } \\
4^{\circ} \text { ESO } \\
1^{\circ} \text { Bachiller } \\
2^{\circ} \text { Bachiller }\end{array}$ & $\begin{array}{l}44 \\
79 \\
31 \\
46\end{array}$ & $\begin{array}{r}77,25 \\
121,12 \\
95,00 \\
91,03\end{array}$ & 3 & 18,814 & ,000 \\
\hline $\begin{array}{l}\text { Motivación } \\
\text { de logro }\end{array}$ & $\begin{array}{l}3^{\circ} \text { ESO } \\
4^{\circ} \text { ESO } \\
1^{\circ} \text { Bachiller } \\
2^{\circ} \text { Bachiller }\end{array}$ & $\begin{array}{l}44 \\
79 \\
31 \\
46\end{array}$ & $\begin{array}{r}84,50 \\
123,06 \\
91,42 \\
83,18\end{array}$ & 3 & 20,744 & ,000 \\
\hline Paz & $\begin{array}{l}3^{\circ} \text { ESO } \\
4^{\circ} \text { ESO } \\
1^{\circ} \text { Bachiller } \\
2^{\circ} \text { Bachiller }\end{array}$ & $\begin{array}{l}44 \\
79 \\
31 \\
46\end{array}$ & $\begin{array}{r}71,19 \\
126,59 \\
85,47 \\
93,85\end{array}$ & 3 & 32,217 & ,000 \\
\hline Prudencia & $\begin{array}{l}3^{\circ} \text { ESO } \\
4^{\circ} \text { ESO } \\
1^{\circ} \text { Bachiller } \\
2^{\circ} \text { Bachiller }\end{array}$ & $\begin{array}{l}44 \\
79 \\
31 \\
46\end{array}$ & $\begin{array}{r}79,28 \\
125,22 \\
88,58 \\
86,38\end{array}$ & 3 & 24,784 & ,000 \\
\hline Religión & $\begin{array}{l}3^{\circ} \text { ESO } \\
4^{\circ} \text { ESO } \\
1^{\circ} \text { Bachiller } \\
2^{\circ} \text { Bachiller }\end{array}$ & $\begin{array}{l}44 \\
79 \\
31 \\
46\end{array}$ & $\begin{array}{r}84,64 \\
124,10 \\
84,98 \\
85,60\end{array}$ & 3 & 21,954 & ,000 \\
\hline Respeto & $\begin{array}{l}3^{\circ} \text { ESO } \\
4^{\circ} \text { ESO } \\
1^{\circ} \text { Bachiller } \\
2^{\circ} \text { Bachiller }\end{array}$ & $\begin{array}{l}44 \\
79 \\
31 \\
46\end{array}$ & $\begin{array}{r}74,93 \\
116,31 \\
98,63 \\
99,07\end{array}$ & 3 & 14,955 & ,002 \\
\hline Solidaridad & $\begin{array}{l}3^{\circ} \text { ESO } \\
4^{\circ} \text { ESO } \\
1^{\circ} \text { Bachiller } \\
2^{\circ} \text { Bachiller }\end{array}$ & $\begin{array}{l}44 \\
79 \\
31 \\
46\end{array}$ & $\begin{array}{r}87,17 \\
127,46 \\
73,53 \\
85,12\end{array}$ & 3 & 30,023 & ,000 \\
\hline Trabajo & $\begin{array}{l}3^{\circ} \text { ESO } \\
4^{\circ} \text { ESO } \\
1^{\circ} \text { Bachiller } \\
2^{\circ} \text { Bachiller }\end{array}$ & $\begin{array}{l}44 \\
79 \\
31 \\
46\end{array}$ & $\begin{array}{r}77,59 \\
116,08 \\
100,95 \\
95,36\end{array}$ & 3 & 13,143 & ,004 \\
\hline
\end{tabular}


$M^{a}$ Inmaculada Herrera Ramírez et al. Los valores de los adolescentes en un centro educativo ...

\begin{tabular}{|c|l|c|c|c|c|c|}
\hline Valores & \multicolumn{1}{|c|}{ Curso } & $\mathbf{N}$ & Rango Promedio & G.L. & $\mathbf{J i}^{\mathbf{2}}$ & $\mathbf{P}$ \\
\hline \multirow{4}{*}{ Verdad } & $3^{\circ}$ ESO & 44 & 63,22 & & & \\
& $\mathbf{4}^{\circ}$ ESO & 79 & $\mathbf{1 2 5 , 7 7}$ & & & \\
& $1^{\circ}$ Bachiller & 31 & 95,98 & & 35,486 & $\mathbf{0 0 0}$ \\
& $2^{\circ}$ Bachiller & 46 & 95,80 & & & \\
\hline
\end{tabular}

$5^{\circ}$. En función del "estatus socio-económico familiar", aplicadas las pruebas "U” de Mann-Whitney y "W" de Wilcoxon, para dos muestras independientes, a los valores de los adolescentes, no se encontraron diferencias estadísticamente significativas en ninguno de los valores analizados.

$6^{\circ}$. En función del "país de nacimiento y nacionalidad", aplicada la prueba de Kruskal Wallis para " $\mathrm{K}$ " muestras independientes a los valores de los adolescentes, no se hallaron diferencias estadísticamente significativas en ninguno de los valores analizados.

$7^{\circ}$. En función de la "cultura/religión" (tabla 5), aplicadas las pruebas "U” de MannWhitney y "W" de Wilcoxon, para dos muestras independientes a los valores de los adolescentes, sólo se han encontrado diferencias estadísticamente significativas en los siguientes valores: humanidad (p. 0,039), igualdad (p. 0,031), liderazgo (p. 0,000), motivación de logro (p. 0,021), religión (p. 0,000), solidaridad (p. 0,014), tolerancia (p. 0,039) y verdad (p. 0,011), obteniendo los mayores rangos promedio los musulmanes; y en: amistad (p. 0,000) y esfuerzo (p. 0,002), los cristianos. El resto de los valores son apreciados por igual.

TABLA 5: Pruebas "U" de Mann-Whitney y "W" de Wilcoxon, para dos muestras independientes de los valores de los adolescentes, con la variable de agrupación "cultura/religión".

\begin{tabular}{|l|l|r|c|r|c|c|c|}
\hline \multicolumn{1}{|c|}{ Valores } & \multicolumn{1}{|c|}{$\begin{array}{c}\text { Cultura/ } \\
\text { Religión }\end{array}$} & $\mathbf{N}$ & $\mathbf{U}$ & $\begin{array}{r}\text { Rango } \\
\text { Promedio }\end{array}$ & $\mathbf{W}$ & $\mathbf{Z}$ & $\mathbf{P}$ \\
\hline Amistad & $\begin{array}{l}\text { Musulmana } \\
\text { Cristiana }\end{array}$ & $\begin{array}{r}120 \\
68\end{array}$ & $2.761,0$ & $\begin{array}{r}83,51 \\
\mathbf{1 1 3 , 9 0}\end{array}$ & $10.021,0$ & 3,718 &, $\mathbf{0 0 0}$ \\
\hline Esfuerzo & $\begin{array}{l}\text { Musulmana } \\
\text { Cristiana }\end{array}$ & $\begin{array}{r}120 \\
68\end{array}$ & $2.957,5$ & $\begin{array}{r}85,15 \\
\mathbf{1 1 1 , 0 1}\end{array}$ & $10.217,5$ & 3,167 &, $\mathbf{0 0 2}$ \\
\hline Humanidad & $\begin{array}{l}\text { Musulmana } \\
\text { Cristiana }\end{array}$ & $\begin{array}{r}120 \\
68\end{array}$ & $3.350,0$ & $\begin{array}{r}\mathbf{1 0 0 , 5 8} \\
83,76\end{array}$ & $5.696,0$ & 2,067 &, $\mathbf{0 3 9}$ \\
\hline Igualdad & $\begin{array}{l}\text { Musulmana } \\
\text { Cristiana }\end{array}$ & $\begin{array}{r}120 \\
68\end{array}$ & $3.324,5$ & $\begin{array}{r}\mathbf{1 0 0 , 8 0} \\
83,39\end{array}$ & $5.670,5$ & 2,158 &, $\mathbf{0 3 1}$ \\
\hline Liderazgo & $\begin{array}{l}\text { Musulmana } \\
\text { Cristiana }\end{array}$ & $\begin{array}{r}120 \\
68\end{array}$ & $2.373,5$ & $\begin{array}{r}\mathbf{1 0 8 , 7 2} \\
69,40\end{array}$ & $4.719,5$ & 4,800 &, $\mathbf{0 0 0}$ \\
\hline $\begin{array}{l}\text { Motivación } \\
\text { de logro }\end{array}$ & $\begin{array}{l}\text { Musulmana } \\
\text { Cristiana }\end{array}$ & $\begin{array}{r}120 \\
68\end{array}$ & $3.266,0$ & $\begin{array}{r}\mathbf{1 0 1 , 2 8} \\
82,53\end{array}$ & $5.612,0$ & 2,301 &, $\mathbf{0 2 1}$ \\
\hline Religión & $\begin{array}{l}\text { Musulmana } \\
\text { Cristiana }\end{array}$ & $\begin{array}{r}120 \\
68\end{array}$ & $2.477,0$ & $\begin{array}{r}\mathbf{1 0 7 , 8 6} \\
70,93\end{array}$ & $4.823,0$ & 4,498 &, $\mathbf{0 0 0}$ \\
\hline
\end{tabular}




\begin{tabular}{|l|l|r|c|c|c|c|c|}
\hline \multicolumn{1}{|c|}{ Valores } & \multicolumn{1}{|c|}{$\begin{array}{c}\text { Cultura/ } \\
\text { Religión }\end{array}$} & $\mathbf{N}$ & $\mathbf{U}$ & $\begin{array}{c}\text { Rango } \\
\text { Promedio }\end{array}$ & $\mathbf{W}$ & $\mathbf{Z}$ & $\mathbf{P}$ \\
\hline Solidaridad & $\begin{array}{l}\text { Musulmana } \\
\text { Cristiana }\end{array}$ & $\begin{array}{r}120 \\
68\end{array}$ & $3.211,0$ & $\begin{array}{r}\mathbf{1 0 1 , 7 4} \\
81,72\end{array}$ & $5.557,0$ & 2,451 &, $\mathbf{0 1 4}$ \\
\hline Tolerancia & $\begin{array}{l}\text { Musulmana } \\
\text { Cristiana }\end{array}$ & $\begin{array}{r}120 \\
68\end{array}$ & $3.344,5$ & $\begin{array}{r}\mathbf{1 0 0 , 6 3} \\
83,68\end{array}$ & $5.690,5$ & 2,069 &, $\mathbf{0 3 9}$ \\
\hline Verdad & $\begin{array}{l}\text { Musulmana } \\
\text { Cristiana }\end{array}$ & $\begin{array}{r}120 \\
68\end{array}$ & $3.191,5$ & $\begin{array}{r}\mathbf{1 0 1 , 9 0} \\
81,43\end{array}$ & $5.537,5$ & 2,551 &, $\mathbf{0 1 1}$ \\
\hline
\end{tabular}

\section{Conclusiones y discusión}

Los resultados obtenidos permitieron extraer las siguientes conclusiones:

$1^{\mathrm{a}}$. Los tres valores más apreciados por los adolescentes de nuestra muestra, en general, se dan en el siguiente orden: igualdad, respeto y espíritu de cambio. En cambio, según Larumbe y Baillo (2001), para los jóvenes universitarios de Zaragoza son: en el caso de los chicos: libertad y amistad, y para las chicas: paz, amistad y libertad; según Elzo (2002), en general, para los jóvenes españoles son: familia, amistad y trabajo; y, según el Instituto de la Juventud (2002), para los jóvenes españoles son: familia, amistad y amor. Aquí se pueden observar pocas similitudes entre los valores más apreciados en la muestra y los resultados obtenidos por los autores citados.

$2^{\mathrm{a}}$. Las adolescentes de la muestra aprecian más la mayoría de los valores, en comparación con los adolescentes. Siendo los tres valores que más aprecian ellas: igualdad, familia y respeto; y, ellos: igualdad, diálogo y espíritu de cambio. Donde, probablemente, se manifiesta la tendencia más familiar y reivindicativa de la mujer adolescente, para sí y para los demás; mientras que el hombre adolescente parece tender más hacia lo social, mostrándose más dispuesto al cambio.

$3^{\mathrm{a}}$. Los adolescentes de $4^{\mathrm{o}}$ de ESO aprecian más la mayoría de los valores, siendo sus tres primeras preferencias: paz, igualdad y diálogo.

$4^{\mathrm{a}}$. En primer lugar, los adolescentes de 16 años aprecian más la mayoría de los valores, siendo sus tres primeras preferencias: amistad, amor y bondad; en segundo lugar, los de 15 años, que aprecian más: democracia, humanidad y liderazgo; y, en tercer lugar, los de 18 años, que se decantan por: esfuerzo, moralidad y tiempo libre y ocio.

$5^{\text {a }}$. Los adolescentes de la muestra, en función de sus culturas/religiones, parecen apreciar los valores de distinta forma. Para los cristianos, los cuatro más apreciados son: familia, respeto, esfuerzo y amistad; y, para los musulmanes: igualdad, verdad, diálogo y bondad. Aproximándose más, en el caso de los adolescentes cristianos, a los resultados obtenidos por Larumbe y Baillo (2001), Elzo (2002) y el Instituto de la Juventud (2002); y, en el caso de los adolescentes de ambas culturas/religiones, con los hallados por Arroyo (2001), en la ciudad de Melilla. 
Lógicamente, estas conclusiones deben ser tenidas en cuenta exclusivamente dentro del contexto, las circunstancias y características de la muestra; es decir, la mayor limitación de esta investigación es precisamente que su poder generalizador queda circunscrito al ámbito donde se ha llevado a cabo y, probablemente, a situaciones y contextos similares.

\section{Referencias bibliográfícas}

Arroyo, R. (2001). "Dependencia e independencia de valores por edad y cultura: Presupuesto del $\mathrm{cu}$ rriculum intercultural". Revista de Investigación Educativa, 19 (1), 153-182.

Elzo, J. (2002). Los valores de los vascos y navarros ante el nuevo milenio: tercera aplicación de la encuesta europea de valores (1990, 1995, 1999). Bilbao: Servicio de Publicaciones de la Universidad de Deusto, 331-382.

Gordon, L. V. (2003). Cuestionario de valores personales (SPV). Madrid: TEA.

Guttman, L. y Lingoes, J. C. (1973). The Guttman-Lingoes nonmetric program series. Arbor: Mathesis Press.

Hartman, R. S. (1959). La estructura del valor: Fundamentos de la axiología cientifica. México: Fondo de Cultura Económica.

Hartman, R. S. (1973). The Hartman Value Profile (HVP). Muskegon, MI.: Research Concepts.

Herrera, M. I. (2007). Los valores de los adolescentes, de sus padres y profesores, en función de que el contexto educativo sea monocultural o pluricultural. Granada: Universidad de Granada.

Hitlin, S. y Piliavin, J. A. (2004). "Values: reviving a dormant concept". Annual Review of Sociology, 30, 359-93.

Instituto de la Juventud (INJUVE) (2002). Sondeo de opinión: Percepción generacional, valores y actitudes, asociacionismo y participación, relaciones sexuales. Madrid: Consejería Técnica de Planificación y Evaluación, Servicio de Documentación y Estudios. Extraído el 12 de Enero de 2007 desde: http://www.laclave.com/docs/documentos/sondeo2002-1a.pdf.

Larumbe, M. A. y Baillo, M. (2001). "Una aproximación a los valores manifestados por los jóvenes universitarios en Zaragoza”. En M. I. Lafuente (Coord.): Los valores en la ciencia y la cultura: Actas del Congreso "Los valores en la ciencia y la cultura". León: Universidad de León, 487-496.

Rokeach, M. (1967). Value survey. Sunnyvale, CA: Halgren Tests.

Rokeach, M. (1973). The nature of human values. New York: Free Press.

Schwartz, S. H. (1992). "Universals in the content and structure of values: Theoretical advances and empirical tests in 20 countries". En M. P. Zanna (Ed.), Advances in experimental social psychology. San Diego: Academic Press, 24, 1-65.

Schwartz, S. H. (1994). "Are there universal aspects in the structure and contents of human values?" Journal of Social Issues, 50, 19-45.

Schwartz, S. H. y Bilsky, W. (1987). "Toward a universal psychological structure of human values". Journal of Personality and Social Psychology, 53 (3), 550-562.

Fecha de recepción: 24-07-06

Fecha de revisión: 30-04-08

Fecha de aceptación: 22-05-08 